\title{
Prevalence of Musculoskeletal Disorder among College Students in Times of COVID-19 Pandemic - An Observational Study
}

\author{
Saumya P. Prajapati ${ }^{1}$, Alpa Purohit ${ }^{2}$ \\ ${ }^{1}$ Post Graduate Physiotherapy Student (MPT in Orthopedics), Ahmedabad Institute of Medical Sciences \\ (AIMS), Ahmedabad, Gujarat, India \\ ${ }^{2}$ Senior Lecturer and Vice Principal, Ahmedabad Institute of Medical Sciences, Ahmedabad, Gujarat, India
}

Corresponding Author: Saumya P. Prajapati

\begin{abstract}
Background: Musculoskeletal disorders are defined as disorders and/or injuries that affect muscles, tendons, skeleton, cartilage, ligaments and nerves. Due to Covid-19, educational institutions were shut down which led to dependency on online classes. Due to decreased physical activity and spending more time on electronic devices in incorrect postures may lead to musculoskeletal disorders.

Purpose of study: To find out the prevalence of musculoskeletal disorders among college students during Covid-19 pandemic to help students know the most prone body areas for musculoskeletal discomfort so as to plan for ergonomic modification and for uplifting musculoskeletal health.

Methodology: A 'Nordic Musculoskeletal Questionnaire' was filled by 250 college students by means of Google forms. Statistical analysis was done using Microsoft Excel 2007.

Results: Among 250 students $64.4 \%$ had at least one musculoskeletal symptom in the previous year. The affection was in Lower back (38\%), Neck (36.4\%), Shoulder (27.20\%), Upper back (22\%), Knee (13.60\%), Wrist (13.20\%), Ankle (12.40\%), Elbow (6.80\%), Hip (6.40\%).

Conclusion: This study concluded that due to increased screen-time on electronic devices during COVID-19 led to high prevalence of musculoskeletal disorders $(64.4 \%)$ in college students. The highest musculoskeletal disorder was found in Lower back region (38\%).
\end{abstract}

Key words: Musculoskeletal disorder, College students, COVID-19

\section{INTRODUCTION}

Musculoskeletal disorder can be defined as "conditions comprise more than 150 diagnoses that affect the locomotor system; that is muscles, bones, joints and associated tissues such as tendons and ligaments, as listed in the International Classification of Diseases" (WHO, 2019). ${ }^{[1]}$ They are caused by forceful or repetitive movements or a poor working posture. Symptoms may include tenderness, aches and pains, tingling, stiffness and swelling.

The COVID-19 pandemic has given rise to a multitude of profound changes in the daily habits of millions of people. Many of these changes are due to lockdown conditions implemented by countries around the world. ${ }^{[2]}$ COVID-19 pandemic has forced people to stay indoors. All educational institutions were shut down and they started online education, this led to dependency on online classes. Thus students were spending more time on laptops and smart phones both to support their academic activities and in their free time. ${ }^{[3]}$

In students receiving online education, additional immobility for extended duration and listening to classes in anti-ergonomic positions may cause pain and musculoskeletal alterations, especially in the upper limb and spine. ${ }^{[3]}$ Computer 
usage that has been more prevalent by distance education may also pose a potential obstacle to student's regular physical activity and exercise. ${ }^{[4]}$ Staying at static position by repeated movements such as using a keyboard, clicking on a mouse, usage of the body in inadequate ergonomic conditions may lead to musculoskeletal disorders. ${ }^{[5]}$ The absence of ergonomic classroom furniture at home may impede the adoption of a healthy posture and may promote the onset of musculoskeletal disorders. $^{[6]}$

Demand for online classes is growing during COVID-19 pandemic but there are no relevant training programs to make student understand or prevent postural problems related to prolonged screen usage. $^{[3]}$ Musculoskeletal pain in students leads to discomfort itself, which may limit the students' daily leisure time, increases psychical stress. Recurrent musculoskeletal pain may impair study performance and affect students' future working capacity and health in their transition from university to professional life. ${ }^{[2]}$ So, here arises a need of this study to know the most prone body area for musculoskeletal disorder so as to plan for ergonomic modification and for uplifting musculoskeletal health of students. The main aim of this study is to find out the prevalence of musculoskeletal disorder among college students in times of COVID19 pandemic.

\section{RESULTS}

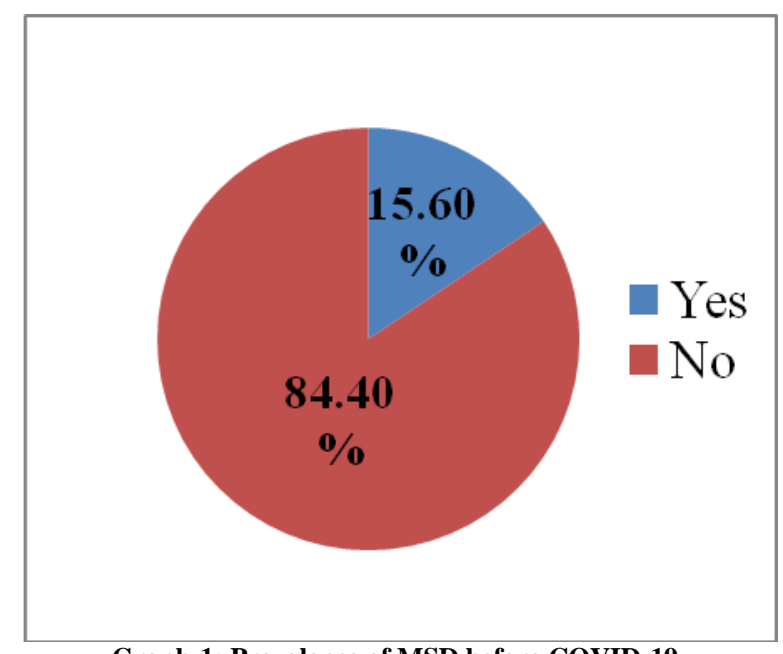

Graph-1: Prevalence of MSD before COVID-19

\section{METHODOLOGY}

A cross-sectional study was conducted from September 2020 to November 2020. 250 undergraduate college students were selected by convenience sampling. A Google form of Nordic Musculoskeletal Questionnaire was created and sent to various undergraduate college students of Ahmedabad and Vadodara city.

Inclusion criteria: Willingness of subject to participate, Both male and female college students, Age group - 18 to 24 years, students who are attending online classes for studies.

Exclusion criteria: Any recent injury/trauma/fracture within 1 year, History of cardiovascular or respiratory problems, On medication or analgesics, Incomplete questionnaire filled by students.

\section{Outcome Measure: Nordic Musculoskeletal Questionnaire (NMQ)}

NMQ interrogates ache, pain or discomfort experienced in the nine body parts (neck, shoulders, back, elbows, wrists/hands, waist, hips/thighs, knees, ankles/feet) during the last 12 months, during last 7 days with binary choice questions (yes or no).

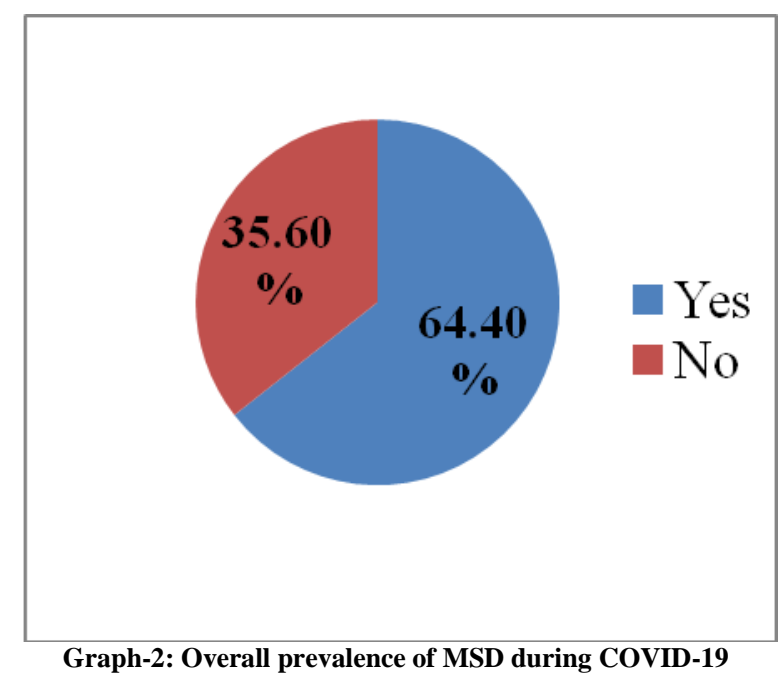


Saumya P. Prajapati et.al. Prevalence of musculoskeletal disorder among college students in times of COVID19 pandemic - an observational study.
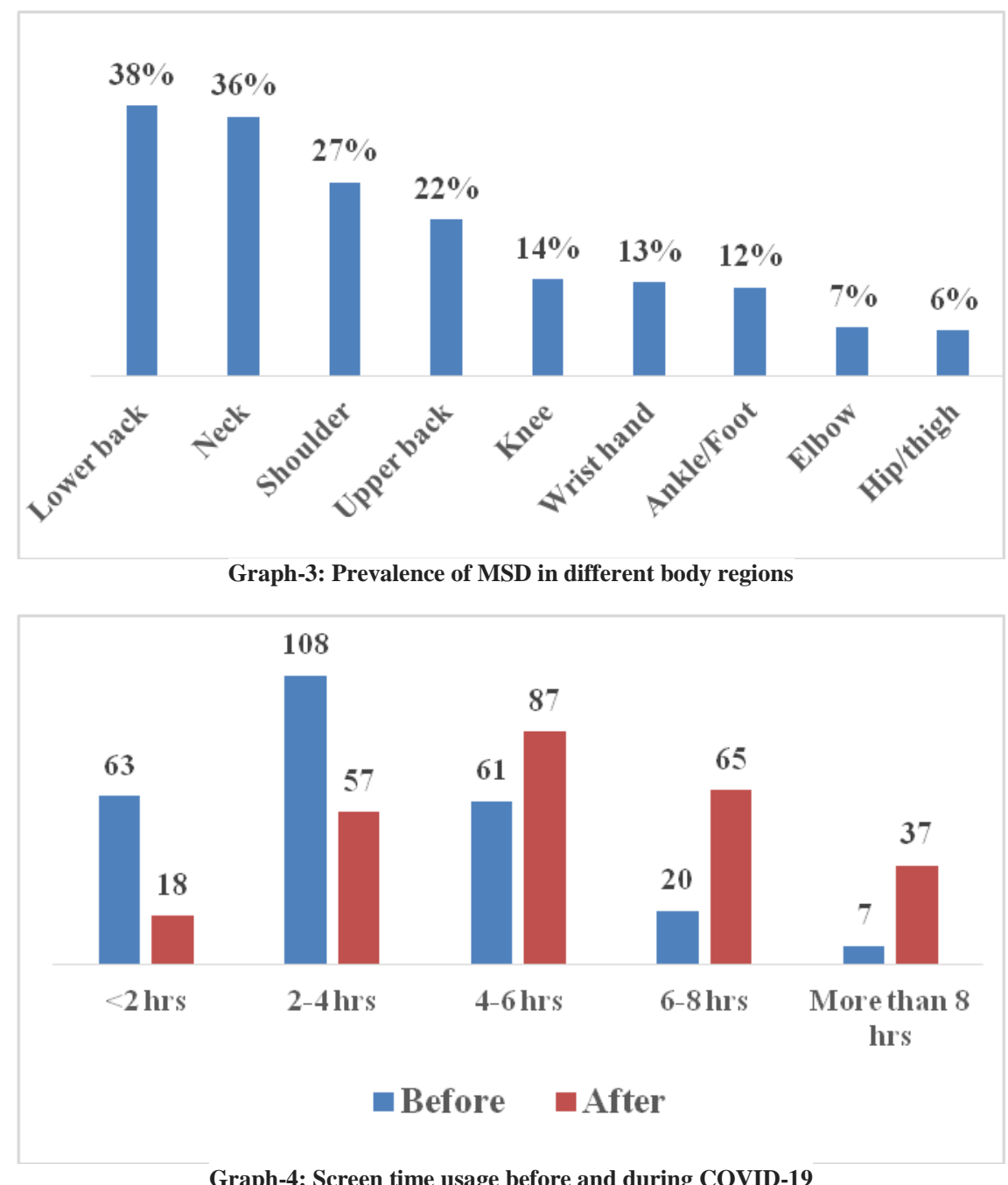

Graph-4: Screen time usage before and during COVID-19

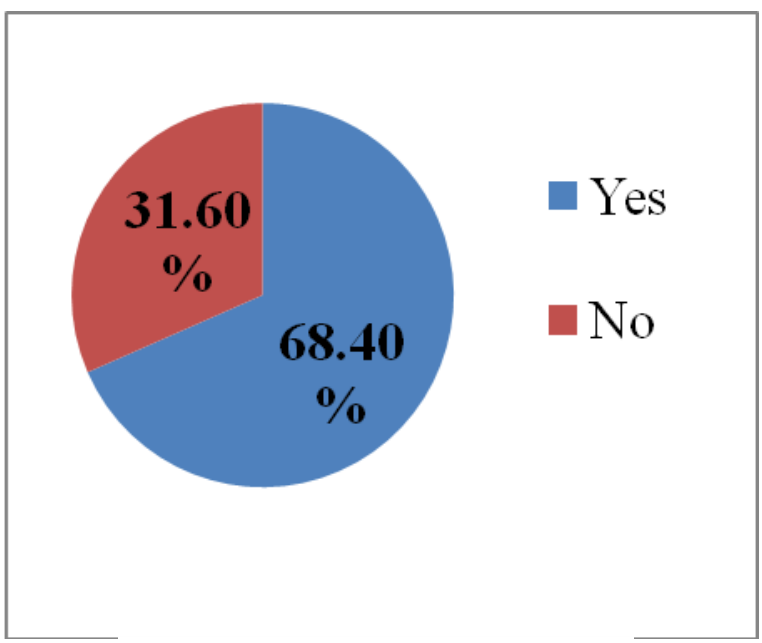

Graph-5: Physical activity before lockdown

Statistical analysis was done using Microsoft Excel 2007. 250 subjects participated in the study. Mean age of the subjects was 19.6 years. Graph-1 illustrates $15.6 \%$ prevalence of MSD before COVID19. Graph-2 demonstrates $64.4 \%$ prevalence of MSD during COVID-19. Graph-3

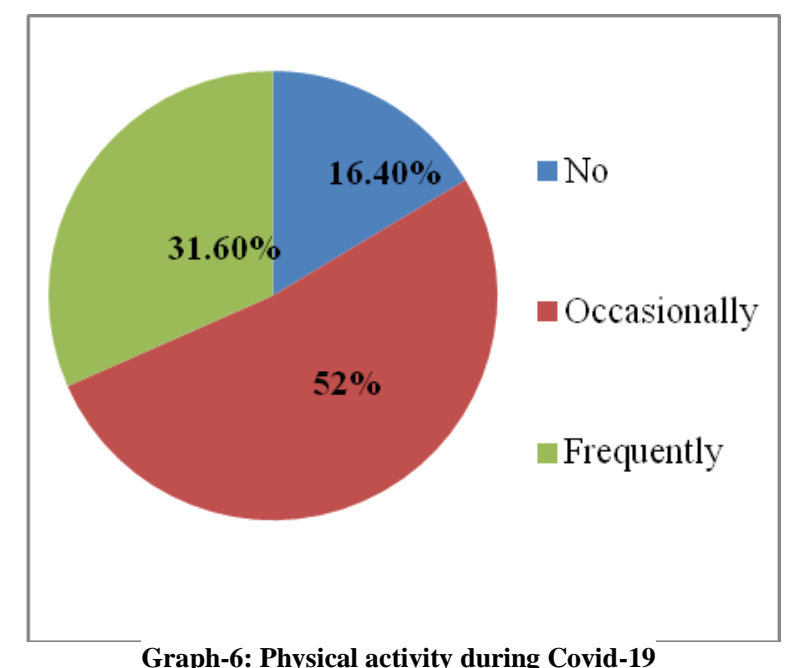

demonstrates prevalence of MSD in different body regions which is highest in lower back (38\%) followed by Neck (36\%) and Shoulder (27\%). Graph-4 illustrates screen-time usage before and after COVID19. Graph-5,6,7 demonstrate physical activity before, during Covid-19 and type of 
Saumya P. Prajapati et.al. Prevalence of musculoskeletal disorder among college students in times of COVID19 pandemic - an observational study.

physical activity respectively, which shows highest number of students did aerobic exercise(42\%). Graph-8 shows $71.2 \%$ population of students has not use specific workplace area for online classes.

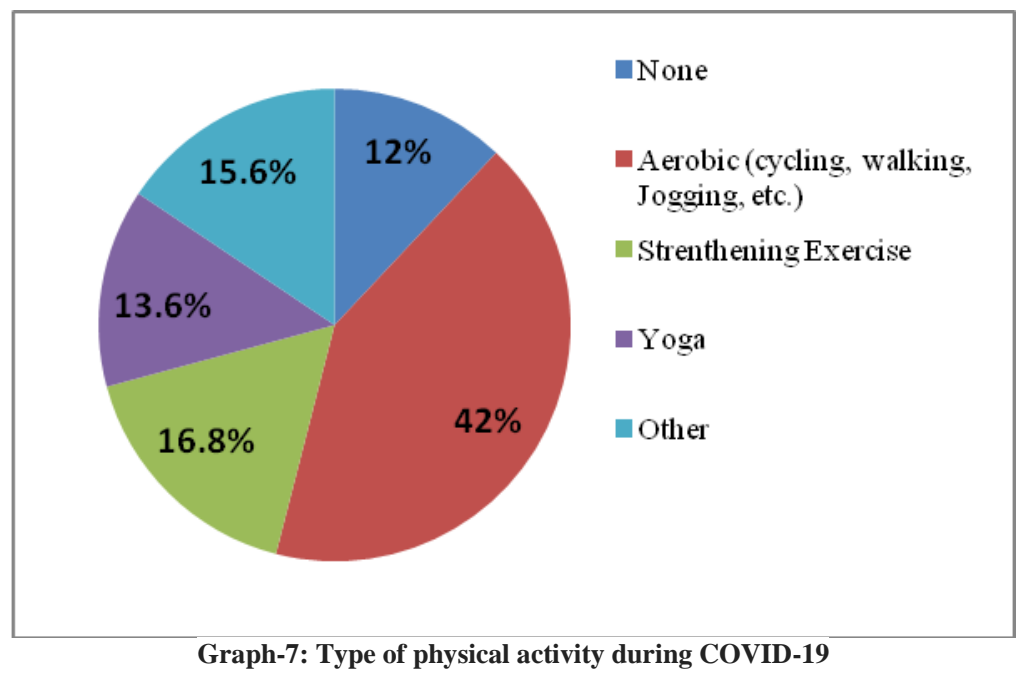

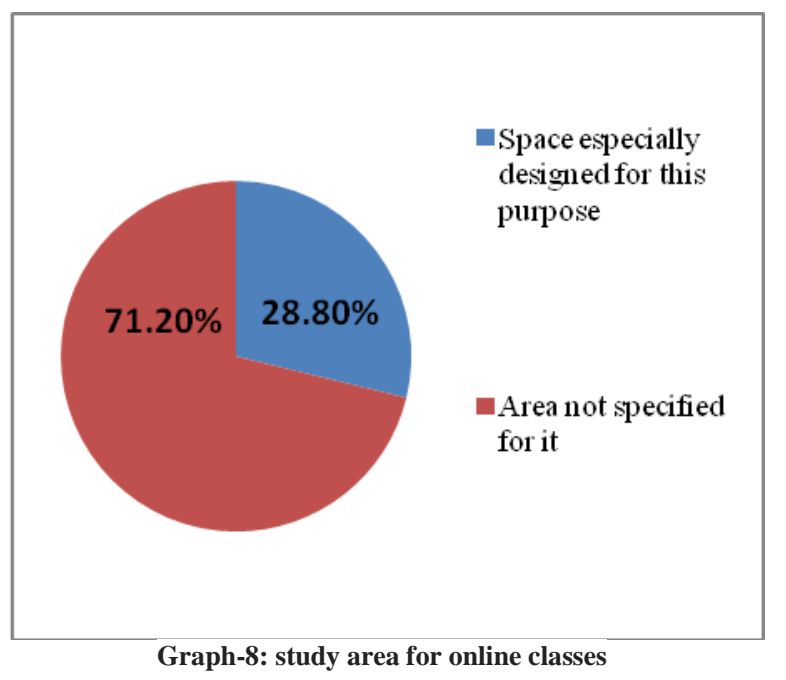

\section{DISCUSSION}

The present study was conducted to see the prevalence of musculoskeletal disorder among 250 college students during COVID-19 pandemic. Result clearly suggests that there is high prevalence of musculoskeletal pain among college students during COVID-19 which has been increased from $15.6 \%$ to $64.4 \%$.

Students' average screen-time usage has increased from average 3 hours before lockdown to 5 hours during lockdown. Pre lockdown around $70 \%$ students were doing exercise, whereas during lockdown around $70 \%$ students were doing physical activity occasionally or not at all. Also, 42\% students were doing aerobic exercise like walking, running, cycling, etc., which might not be possible in the initial phase of lockdown. $71.2 \%$ population of students, has not use any specific ergonomically designed area to attend online classes, which can also adversely affect the students' posture.

Sometimes students did not have chair at home that is ergonomically standard and students often sat with slouched posture, it will reduce the muscular effort that is required to maintain a seated position will cause the ligaments and muscles which support the spine happens the increasing the tension. So, seated position is possible to cause the upper and lower back pain. [3] Prolonged static contraction of the skeletal muscles of the back causes impairment of the blood flow to the muscles (ischemia), together with oedema and accumulation of the waste metabolites which will trigger the pain spasm. ${ }^{[5]}$

As stated by Burdorf et al., a sustained sedentary job in a forced nonneutral trunk posture is a risk factor for LBP. ${ }^{[7]}$ Due to low-grade activation of lumbar muscles while sitting, the load is conducted by passive structures such as ligaments and intervertebral discs. Because of the viscoelasticity of these structures and deactivation of lumbar muscles, the lumbar 
spine may be predisposed to deconditioning and LBP. ${ }^{[8]}$

Forward head posture is a known cause which puts lot of pressure on cervical and lumbosacral spine, intervertebral disc and the pain resulting from the respective nerve territories. Fixed postures of neck while focusing on the monitor probably caused severe pain in the neck and shoulder muscles. The persistent pain in the shoulder area is also attributable to static loading or isometric contraction of muscles of neck, shoulder and arms in order to maintain the position of function. ${ }^{[3]}$ During working activities in front of the computer, upper trapezius muscle was the most exerted muscle that leads to the generation of internal forces within the body in which it will increase the muscle tension and pain in the shoulder and neck. ${ }^{[5]}$

Wrist and hand pain may be due to overuse of touch screen devices. Repetitive typing or clicking on mouse can cause micro trauma in carpal tunnels. ${ }^{[3]}$

Erik and Smith (2011) confirmed by the systemic review that prevalence of MSD ranges between $39 \%$ and $95 \%$ in which most prevalent body sites to be the neck, back and upper limbs. ${ }^{[9]}$

The important limitation of this study is small sample size. Large sample size would be more helpful. According to screen-time usage and type of electronic device wise prevalence of musculoskeletal pain can be carried out in future. Musculoskeletal disorder has a variety of simultaneous influences that need to be accounted for in future studies.

\section{Clinical implications:}

- Teachers, parents and students must be trained in fundamentals of workstation ergonomics.

- Students should advise for deskbound stretches.

- Students should be encouraged to have a frequent micro breaks from their sitting.

- Students with musculoskeletal disorders should be treated according to their condition.

\section{CONCLUSION}

This study concluded that due to increased screen-time on electronic devices during COVID-19 led to high prevalence of musculo-skeletal disorders $(64.4 \%)$ in college students. The highest prevalence was found in Lower back region (38\%).

\section{ACKNOWLEDGMENTS}

I am very grateful to my guide who supported me constantly throughout the study. I am very thankful to all the subjects who participated in this study to make it worth.

\section{Conflict of Interest: None}

\section{Source of Funding: None}

\section{REFERENCES}

1. World Health Organization (WHO) (2020) Musculoskeletal Conditions. World Health Organization. Geneva, Switzerland

2. Leirós-Rodríguez R, Rodríguez-Nogueira Ó, Pinto-Carral A, Álvarez-Álvarez M, Galán-Martín MÁ, Montero-Cuadrado F, Benítez-Andrades JA. Musculoskeletal Pain and Non-Classroom Teaching in Times of the COVID-19 Pandemic: Analysis of the Impact on Students from Two Spanish Universities. Journal of Clinical Medicine. 2020 Dec;9(12):4053.

3. Choudhary MS, Choudary AB, Jamal S, Kumar R, Jamal S. The impact of ergonomics on children studying online during COVID-19 lockdown. J Adv Sport Phys Edu August. 2020;3(8):117-20.

4. TİGLİ A, ALTINTAŞ A, AYTAR A. Effects of posture and ergonomics training for students receiving distance education during the covid-19 pandemic on musculoskeletal pain, exercise behavior decision-making balance, and physical activity level. Journal of Exercise Therapy and Rehabilitation. 2020;7(2):137-44.

5. Bachtiar F, Maharani FT, Utari D. Musculoskeletal Disorder of Workers During Work From Home on Covid-19 Pandemic: A Descriptive Study. In International Conference of Health Development. Covid-19 and the Role of Healthcare Workers in the Industrial Era 
Saumya P. Prajapati et.al. Prevalence of musculoskeletal disorder among college students in times of COVID19 pandemic - an observational study.

(ICHD 2020) 2020 Nov 25 (pp. 153-160). Atlantis Press.

6. Moretti A, Menna F, Aulicino M, Paoletta M, Liguori S, Iolascon G. Characterization of home working population during COVID-19 emergency: a cross-sectional analysis. International journal of environmental research and public health. 2020 Jan;17(17):6284.

7. Burdorf, A.; Naaktgeboren, B.; de Groot, H.C. Occupational risk factors for low back pain among sedentary workers. J. Occup. Med. 1993, 35, 1213-1220

8. Mörl, F.; Bradl, I. Lumbar posture and muscular activity while sitting during office work. J. Electromyogr. Kinesiol. 2013, 23, 362-1368.

9. Erick PN, Smith DR. A systematic review of musculoskeletal disorders among school teachers. BMC musculoskeletal disorders. 2011 Dec;12(1):1-1.

How to cite this article: Prajapati SP, Purohit A. Prevalence of musculoskeletal disorder among college students in times of COVID-19 pandemic - an observational study. Int $J$ Health Sci Res. 2021; 11(10): 214-219. DOI: https:// doi.org/10.52403/ijhsr.20211028 